\title{
Study on Order Analysis for Condition Monitoring Wind Turbine Gearbox
}

\author{
FENG Yanhui ${ }^{\star}$, LI Jiawei*, QIU Yingning*, YANG Wenxian', INFIELD David \\ *School of Energy and Power Engineering,Nanjing University of Science and Technology, China. \\ †School of Marine Science and Technology, Newcastle University, UK. \\ $\uparrow \uparrow$ Department of Electronic and Electrical Engineering, The University of Strathclyde, UK. \\ yanhui.feng@njust.edu.cn, fongyanfai@hotmail.com
}

Keywords: Condition monitoring, order analysis, angular sampling, planetary gearbox, wind turbine

\begin{abstract}
A complex 3-stage gearbox design, in which one planetary gear set at first stage and two sets of parallel gears at second and third stage, is conventionally applied on the large wind turbine configuration with Doubly Fed Induction Generator. In this variable-speed wind turbine, it is hard to directly apply conventional frequency analysis on condition monitoring of the gearbox planetary stage. Proper signal processing and analysis become crucial here for interpreting the conditions of gears and bearings. In this paper, order analysis is studied for condition monitoring the planetary stage of wind turbine gearbox. The approach takes advantage of angular resampling to achieve cyclo-stationary vibration signals and lessen the effects due to speed changes. One key element in the scheme is taking angular information to enable resampling the time-domain signals into angular-domain signals. A vibration signal model for gearbox planetary stage is firstly described, and the scheme is then tested on simulated signals to achieve fine frequency resolution. Time synchronous averaging is applied as a pre-processing technique to enhance the Signal to Noise Ratio (SNR) in order spectrum.
\end{abstract}

\section{Introduction}

Survey by Global Wind Energy Council shows total globally installed wind capacity has reached $318 \mathrm{GW}$ by end of 2013, has doubled from the total installed capacity of $159 \mathrm{GW}$ in 2009. This means that more than 200,000 large wind turbines of different sizes, makes and ages are operating in the world today. Deployment of larger scale wind turbine systems, particularly offshore, requires more optimised Operation \& Maintenance strategies to ensure system reliability.

Nowadays, planetary gearbox or epicyclic gearbox is one of the critical mechanisms in wind power generation. Modern wind industry has ever experienced severe failure problems of gearbox. Wind farm operators are concerning on gearbox reliability due to its complex repair procedures, high replacement costs and long downtimes leading to revenue losses. It is important to perform effective condition monitoring and fault diagnosis on wind turbine gearbox to improve reliability and reduce downtime. Wind turbine usually requires planetary gearbox with high transmission ratio and compact design. It transmits the rotating power of high torque from input shaft to the high speed end which is coupled with generator rotor. Please see Figure 1 for a typical 3 -stage planetary gearbox. The planetary gear set is comprised of an outer ring, three planetary gears to share load, and a sun gear. The planetary gear stage, coupled with low speed shaft to take on wind loading, is comprised of planetary gears in a planet carrier coaxial with a sun gear and a ring gear [1]. In this design, vibration and transmission path at the planetary stage are much more complicated than those at two parallel stages. Constrained by limited mounting space and hardware investment, only 1-2 accelerometers is normally mounted at the planetary stage to measure vibration. On the other hand, the rotating speed of wind turbine gearbox input shaft is slow at less than $20 \mathrm{rpm}$, and time-varying subject to wind speed variations. It causes the characteristic frequencies of components at planetary stage are very low and unstable, which can easily affected by frequency components of background noise close to DC.

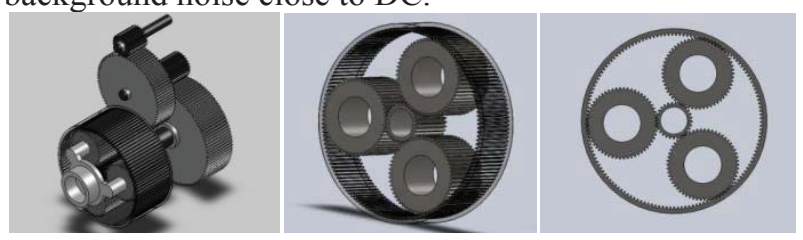

Figure 1. Schematic diagrams of 3-stage wind turbine gearbox and the planetary gear set

In wind industry, most of commercially available condition monitoring systems use time domain or spectral analysis of vibration signals for gearbox fault diagnosis [2]. By studying vibration monitoring, some research works reported the cepstrum and narrowband phase modulation signal were effective methods for diagnosing gear tooth problems, whereas bearing envelope analysis could confidently detect most of the bearing-related failures [3]. The rotating speed of wind turbine gearbox is decided by the wind speed in which gust, turbulence and ramp are main components. Therefore, it is not easy to properly interpret vibration signals using traditional analysis either in frequency domain or in time domain when turbine gearbox operates subject to wind intermittence and randomness. In addition, there exist some methods in the regime of mechanical engineering to deal with fault diagnostic challenges in variable-speed machinery, particularly for bearings. For example, Zhao et al. proposed a technique, combining tacholess order tracking and envelope order spectrum to recover the bearing characteristic frequencies in the order domain under varying speeds [4]. 
Rémond, Antoni and Randall noted in [5] that mechanical models should be considered with a new way of thinking as soon as differential equations are rephrased in the angular domain.

In this study, we focus on the planetary gearbox fault diagnosis in wind turbine. At first, we describe the vibration signals models of wind turbine gearbox and revisit the concept of order analysis. Based on the numerical simulation technique, we compare the frequency analysis (i.e. FFT) and order analysis of vibration signals assuming gearbox operates at fix speed or at variable speed. Time synchronous averaging is then applied to enhance the SNR.

\section{Defect frequencies in planetary gear set}

The defect frequencies found the basis of vibration diagnosis for wind turbine. In a general case, we define the following notations:

\begin{tabular}{|c|l|}
\hline$F_{p}$ & rotating frequency of planetary gear \\
\hline$F_{c}$ & rotating frequency of carrier \\
\hline$F_{S}$ & rotating frequency of sun gear \\
\hline$Z_{p}$ & the number of teeth on planetary gear \\
\hline$Z_{S}$ & the number of teeth on sun gear \\
\hline$Z_{r}$ & the number of teeth on ring gear \\
\hline$n_{p}$ & the number of planetary gears \\
\hline$F_{\text {mesh }}$ & gear mesh frequency \\
\hline$F_{\text {p.mes }}$ & planetary gear mesh frequency \\
\hline$F_{\text {planet }}$ & local defect frequency of planetary gear \\
\hline$F_{\text {sun }}$ & local defect frequency of sun gear \\
\hline$F_{\text {ring }}$ & local defect frequency of ring gear \\
\hline
\end{tabular}

Since the planetary gears mesh with ring gear and sun gear simultaneously, the fundamental mesh frequency $F_{\text {mesh }}$ is calculated as:

$F_{\text {mes } h}=\left(F_{s}-F_{c}\right) Z_{s}=\left(F_{p}-F_{c}\right) Z_{p}=F_{c} Z_{r}$.

The above only considers one planetary gear, for $n_{p}$ number of planetary gears, the mesh frequency $F_{\text {p.mes } h}$ is expressed as: $F_{\text {p.mesh }}=n_{p} \cdot F_{\text {mesh }}$.

When a local defect occurs on the ring gear, its defect frequency $F_{\text {ring }}$ is relevant to the number of planetary gears $n_{p}$ and rotating frequency $\mathrm{d}$ of carrier $F_{c}$, such that

$F_{\text {ring }}=n_{p} \cdot F_{c}$.

Combined with the equation (1), the defect frequency can be written as

$F_{\text {ring }}=n_{p} \cdot F_{s} /\left(2+2 Z_{p} / Z_{s}\right)=n_{p} \cdot F_{s} /\left(1+Z_{r} / Z_{s}\right)$.

The sun gear only meshes with planetary gears, its defect frequency $F_{\text {sun }}$, such that:

$F_{\text {sun }}=n_{p}\left(F_{s}-F_{c}\right)$.

A planetary gear interacts with ring gear and sun gear in one revolution, the defect frequency $F_{\text {planet }}$ is:

$F_{\text {planet }}=F_{p}=F_{\text {mesh }} / Z_{p}$.

Assuming the same dynamic response for both interactions, the defect frequency is twice of the above:

$F_{\text {planet }}=2 F_{\text {mesh }} / Z_{p}$.

Combined with the equation (1), all of the defect frequencies in planetary gear set can be written as a linear function of the carrier rotating frequencies $F_{c}$, teeth and gear numbers. Since the carrier is coupled with main shaft in wind turbine, its rotating frequency is equal to that of the rotor.

\section{Methodology}

\subsection{Vibration signals modelling}

When a faulty gear meshes with other parts in a planetary gear, the vibration signal can be modelled as an amplitude modulation and frequency modulation process [6]:

$x(t)=h(t) \sum_{k=0}^{\infty} a_{k}(t) \cos \left[2 \pi k f F_{\text {mes } h} t+b_{k}(t)+\theta_{k}\right]$, in which $x(t)$ is vibration response amplitude, $h(t)$ models the effect of vibration transfer path, $F_{\text {mes }}$ mesh frequency, $a_{k}(t)$ amplitude modulation function, $b_{k}(t)$ frequency modulation function, $\theta_{k}$ initial phase of amplitude modulation, $\mathrm{k}$ is the integer.

If only fundamental frequencies (gear mesh frequency and local defect frequency) are considered, the model is simplified as

$x(t)=\left[1+A \cos \left(2 \pi f_{g} t+\emptyset\right)\right] \cdot \cos \left[2 \pi F_{\text {mesh }} t+B \sin \left(2 \pi f_{g} t+\varphi\right)+\theta\right]$ in which $\mathrm{A}, \mathrm{B}$ are the magnitudes, $f_{g}$ is the defect frequency.

\subsection{Angular re-sampling}

In variable-speed rotating machinery, vibration signals are non-stationary due to speed alteration. Order analysis is a useful signal processing technique in this case. By resampling the non-stationary vibration signals, it is possible to reconstruct cyclo-stationary vibration signals in angular domain, to avoid the mismatch between angle and time information. One can further process the re-sampled vibration signals to achieve order spectra via Fourier transform, and to improve SNR via synchronous averaging approach. In addition to the vibration signals in time domain, it is essential to collect or synthesise the angular impulses with time stamp for performing the order analysis. Please refer to Figure 2.

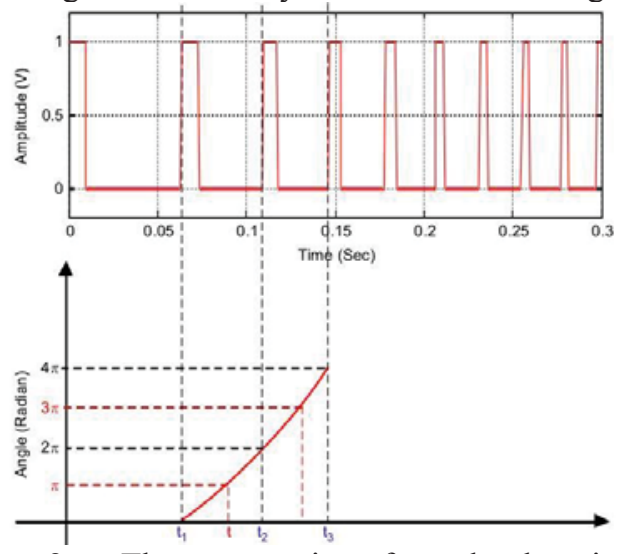

Figure 2. The construction of angular domain [7]

\section{Results}

When a local defect occurs on sun gear, its meshing point between sun gear and planetary gear will rotate by the sun gear. The magnitude of measured vibration signal depends on the distance between meshing point and transducer mounting 
point. The detection of sun gear defect is one of most challenge works in wind industry. The rotation of sun gear also generates amplitude modulation on vibration signals. According to the model in (9), the measured vibration signal model is

$x(t)=\left[1-\cos \left(2 \pi F_{s} t\right)\right]\left[1+A \cos \left(2 \pi F_{\text {sun }} t+\emptyset\right)\right]$.

$\cos \left(2 \pi F_{\text {mes } h} t+B \sin \left(2 \pi F_{\text {sun }} t+\varphi\right)+\theta\right)$,

in which $\left[1-\cos \left(2 \pi F_{s} t\right)\right]$ is the term of amplitude modulation caused by rotation of sun gear.

Let initial phase as $0, \mathrm{~A}$ and $\mathrm{B}$ magnitudes as 1 , gearbox operating at fixed speed such that $F_{S}=15 \mathrm{~Hz}, F_{\text {sun }}=$ $40 \mathrm{~Hz}, F_{\text {mes } h}=180 \mathrm{~Hz}$, sampling rate is $20480 \mathrm{~Hz}$, Figure 3 and Figure 4 show the simulated vibration signals of sun gear in time domain and in frequency domain.
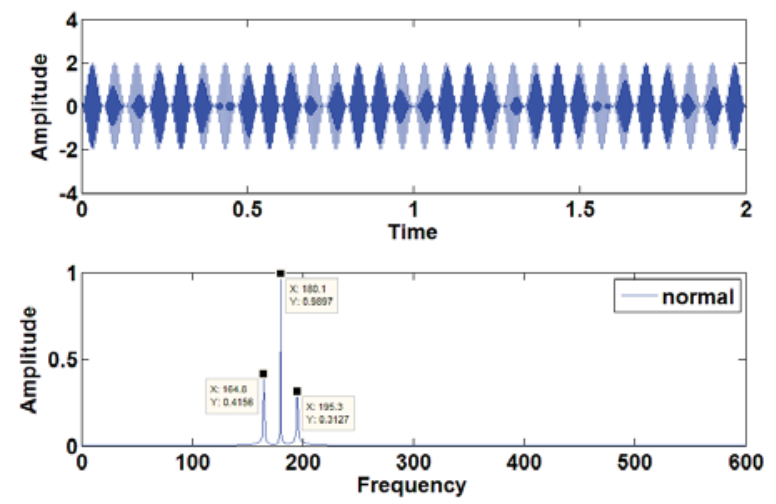

Figure 3. Vibration signal of sun gear without local defect in time domain(top) and frequency domain(bottom)
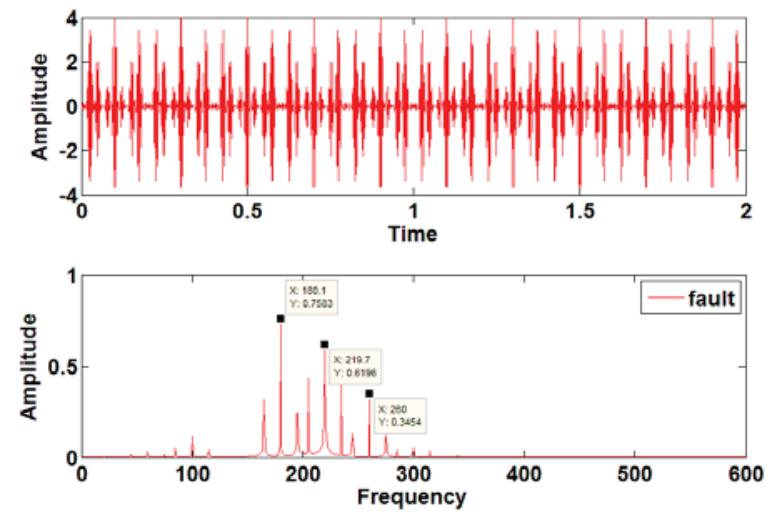

Figure 4. Vibration signal of sun gear with local defect in time domain(top) and frequency domain(bottom)

Figure 3 shows only gear mesh frequency $F_{\text {mes } h}$ (at $180.1 \mathrm{~Hz}$ ) and the sidebands $F_{\text {mes }} \pm F_{S}$ (at $195.3 \mathrm{~Hz}, 164.8 \mathrm{~Hz}$ ) appear in the vibration spectra of sun gear without local defect. Once a local defect occurs, Figure 4 shows that $F_{\text {mesh }}+F_{\text {sun }}$ (at $219.7 \mathrm{~Hz}$ ) along with the sidebands $F_{\text {mes } h}+F_{\text {sun }} \pm F_{s}$, $F_{\text {mesh }} \pm 2 F_{\text {sun }}$ (at $100 \mathrm{~Hz}, 260 \mathrm{~Hz}$ ) along with the sidebands $F_{\text {mes } h} \pm 2 F_{\text {sun }} \pm F_{s}$, and the high order harmonics appear in the spectra. It is then possible to identify local defect directly from vibration spectra. However, the frequency spectra are quite different if gearbox operates at variable speed. In this situation, every frequency changes following change of speed. Here let $Z_{r}=103, Z_{s}=20, n_{p}=3$, gearbox operating at variable speed $F_{c}=t$, from equation(1) $F_{s}=(1+$ $\left.Z_{r} / Z_{s}\right) F_{c}=6.15 t$, such that $F_{\text {mes } h}=103 t, F_{\text {sun }}=15.45 t$,
Figure 5 and Figure 6 show the simulated vibration signals of sun gear when gearbox operates at variable speed. Comparing to the vibration spectra of gearbox at fixed speed, we cannot identify characteristic frequencies from the smeared spectra.
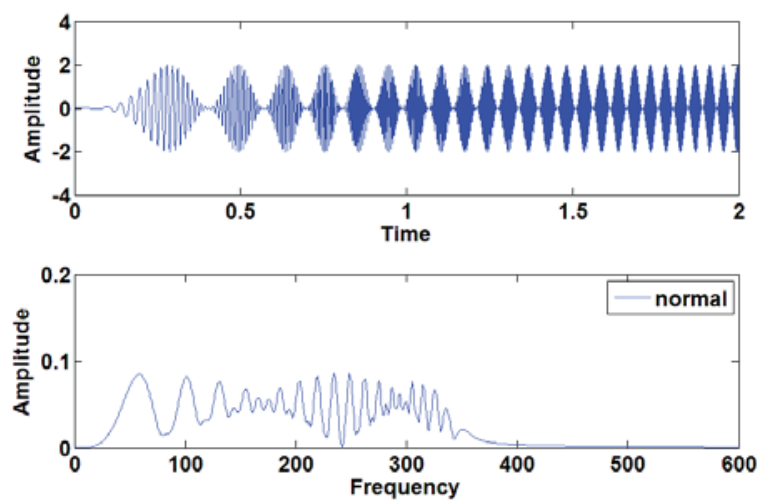

Figure 5. Vibration signal of sun gear without local defect under variable-speed operation in time domain(top) and frequency domain(bottom)
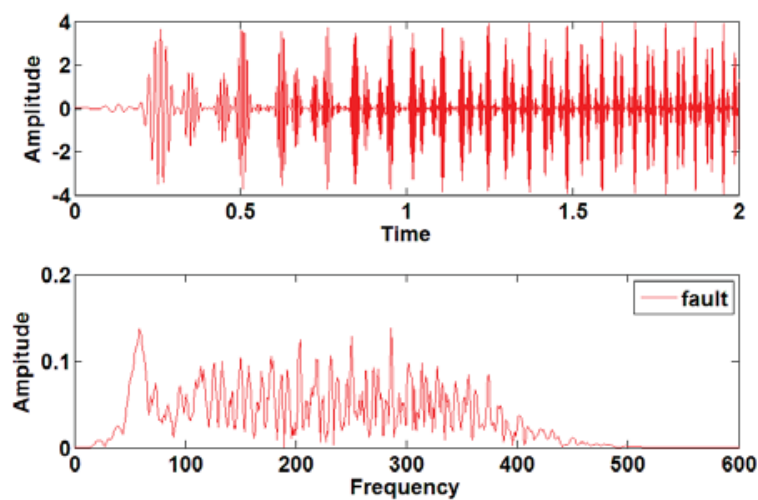

Figure 6. Vibration signal of sun gear with local defect under variable-speed operation in time domain(top) and frequency domain(bottom)
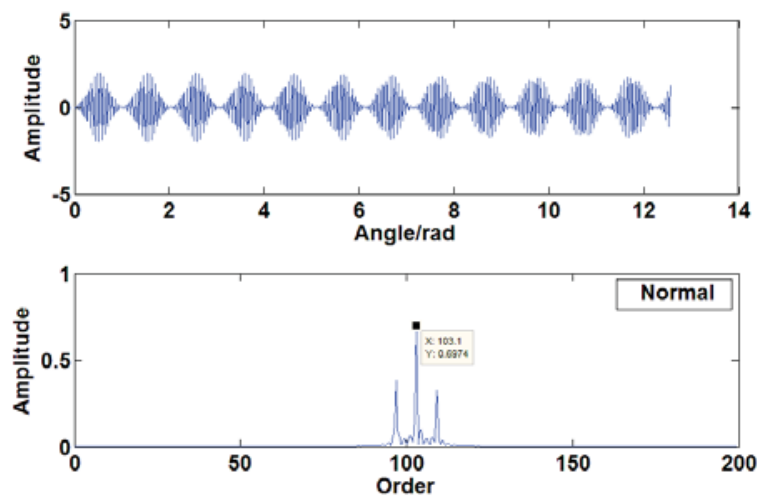

Figure 7. Re-sampled vibration signals of sun gear without defect in angular domain(top) and in order spectrum(bottom) at variable speed.

To enable the fault diagnosis, we then apply the angular resampling (400 samples/2pi) and order analysis on the signals. The non-stationary vibration signals is firstly re-sampled to achieve cyclo-stationary signals in angular domain, then transformed into order spectrum with regard to the rotation of planet carrier. Figure $7 \&$ Figure 8 show the re-sampled 
vibration signals of sun gear in angular domain and in order spectrum at variable speed. $F_{\text {mes } h}$ is 103 times of $F_{c}$, sun gear rotating frequency $F_{s}$ is 6.15 times of $F_{c}, F_{\text {sun }}$ is 15.45 times of $F_{c}$. We can easily observe the mesh order 103.1 and the sidebands $F_{\text {mes } h} \pm F_{s}$ from Figure 7 . Once a local defect occurs, Figure 8 shows that $F_{\text {mes }}+F_{\text {sun }}$ (at the order 118.4), $F_{\text {mes } h}+2 F_{\text {sun }}$ (at the order 134) along with sidebands.
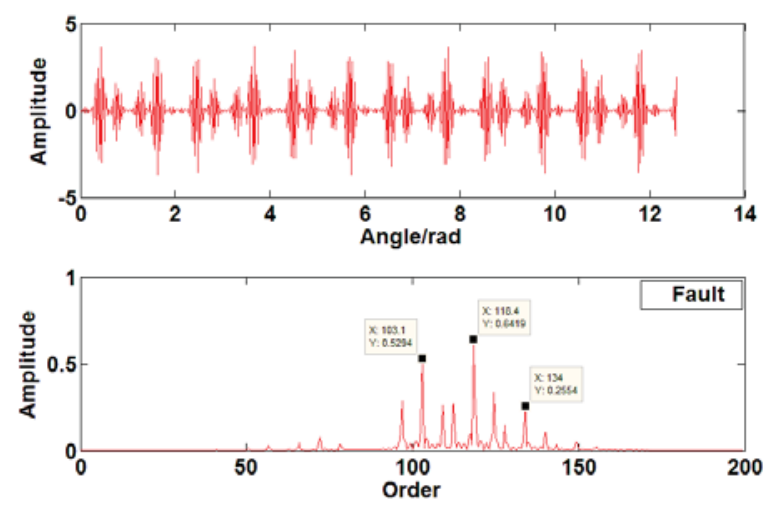

Figure 8. Re-sampled vibration signals of sun gear with defect in angular domain(top) and in order spectrum(bottom) at variable speed.

When background noise increases in vibration signals, in general, the characteristics in order spectrum are inevitably masked by noise. Please refer to Figure 9.
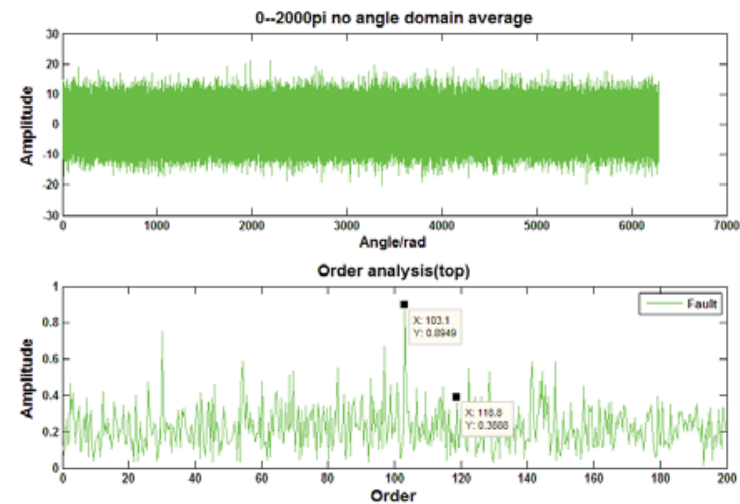

Figure 9. Order spectrum without synchronous averaging (raw signal length=2000pi, SNR=1:5)
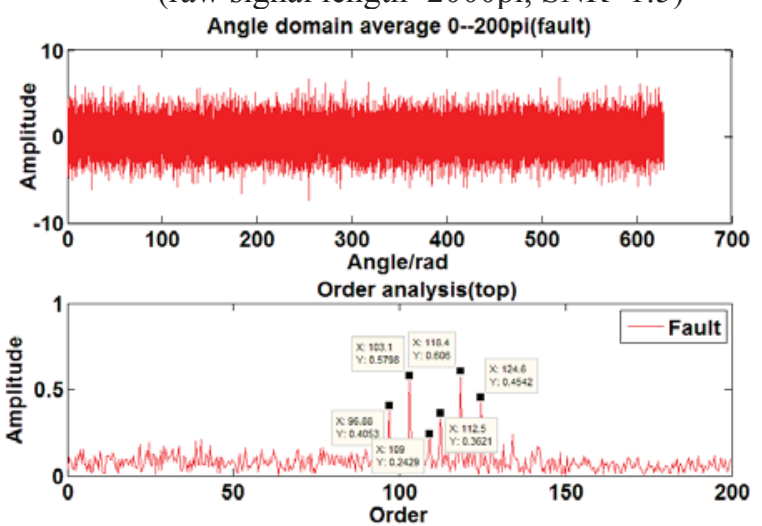

Figure 10. Order spectrum with synchronous averaging (raw signal length=200pi, SNR=1:5)

By use of the time synchronous averaging technique, we can enhance signals synchronised in angular domain, and reduce those noise in asynchronous phase. Figure 10 shows the order spectrum with synchronous averaging for the raw signal sample length is 200pi in angle and SNR 1:5. Even with a much longer sample length 2000pi, for comparison, characteristic orders in Figure 9 are much invisible if without applying the synchronous averaging technique.

\section{Conclusions}

- Vibration signal model of planetary gear set is a complicated frequency modulation and amplitude modulation process.

- Numerical simulations show angular re-sampling and order analysis are capable to extract characteristic orders (i.e. the number of times of planet carrier rotating frequency) for diagnosing wind turbine gearbox which operates at variable speed.

- Time synchronous averaging, as a pre-processing technique, is powerful for enhancing Signal to Noise Ratio in order spectrum.

\section{Acknowledgement}

This work is funded by Jiangsu Top Six Talent Summit Fund (ZBZZ-045) and Jiangsu Province Natural Science Fund (BK 2013135).

\section{References}

[1] Yanhui Feng, Yingning Qiu, Christopher J. Crabtree, Hui Long and Peter J. Tavner. Monitoring wind turbine gearboxes. Wind Energy 2013, vol. 16:728-740, DOI: 10.1002/we. 1521 .

[2] Wenxian Yang, Peter J. Tavner, Christopher J. Crabtree, Y. Feng and Y. Qiu. Wind turbine condition monitoring: technical and commercial challenges. Wind Energy 2014 vol. 17: 673-693. doi: 10.1002/we.1508.

[3] David Siegel, Wenyu Zhao, Edzel Lapira, Mohamed AbuAli and Jay Lee. A comparative study on vibrationbased condition monitoring algorithms for wind turbine drive trains. Wind Energy 2014, vol. 17: 695-714. doi: 10.1002/we. 1585 .

[4] Ming Zhao, Jing Lin, Xiaoqiang $\mathrm{Xu}$ and Yaguo Lei. Tacholess Envelope Order Analysis and Its Application to Fault Detection of Rolling Element Bearings with Varying Speeds. Sensors 2013, 13: 10856-10875.

[5] D. Rémond, J. Antoni, R.B. Randall. Instantaneous Angular Speed (IAS) processing and related angular applications. Mechanical Systems and Signal Processing. 45 (2014) 24-27.

[6] Zhipeng Feng, Ming J. Zuo, Jian Qu, Tao Tian, Zhiliang Liu. Joint amplitude and frequency demodulation analysis based on local mean decomposition for fault diagnosis of planetary gearboxes. Mechanical Systems and Signal Processing 40 (2013) 56-75.

[7] Jinjiang Wang, Robert X. Gao, Ruqiang Yan. Multi-scale enveloping order spectrogram for rotating machine health diagnosis. Mechanical Systems and Signal Processing. 46 (2014) 28-44. 